\title{
Isolated chordal shortening: a novel mechanism of functional mitral regurgitation
}

\author{
Evann E. Eisenberg ${ }^{1}$, Ayesha Salahuddin ${ }^{1}$, Neena Passi ${ }^{1}$, Waddy Gonzalez ${ }^{1}$, Daniel Schwartz ${ }^{1}$, \\ Wilbert S. Aronow ${ }^{1}$, Daniel M. Spevack ${ }^{1,2}$
}

\begin{abstract}
${ }^{1}$ Department of Medicine, Cardiology Division, Montefiore Medical Centre and Albert Einstein College of Medicine, Bronx, New York, USA

2Department of Cardiology, Westchester Medical Centre and the Department

of Medicine, New York Medical College, Valhalla, New York, USA
\end{abstract}

Submitted: 14 March 2020

Accepted: 21 March 2020

Arch Med Sci Aging 2020; 3: e7-e12

DOI: https://doi.org/10.5114/amsa.2020.94952

Copyright (c) 2020 Termedia \& Banach

\section{Abstract}

Introduction: Mitral regurgitation (MR) that occurs in the absence of primary leaflet disease is known as functional MR. MR that occurs in the absence of primary leaflet disease is known as functional MR. Functional MR generally results from left ventricular (LV) enlargement, altered geometry of the papillary muscles, and/or dilatation of the mitral valve annulus. At our institution, we noted a group of patients with surgical MR, who did not have either primary leaflet disease or obvious alteration in LV geometry. We present a cohort of patients with MR secondary to isolated chordal shortening. Material and methods: The study population consisted of subjects with normal mitral leaflet appearance, left ventricular size and function, and mitral annular dimension by echocardiography. Valve morphology and appearance were confirmed by inspection during surgery and by pathological examination when available. Mitral valve tethering parameters were compared to sample subjects with normal valves, and to sample subjects with severe ischaemic functional MR. Both control groups were matched to the study cohort both by age, sex, and body surface area.

Results: Ten subjects met the inclusion criteria. On surgical inspection, chordal shortening or restriction was reported in six and chordal thickening or fibrosis was mentioned in two subjects. Compared to normal controls, the study group had shorter chordae, increased mitral tenting height, and smaller mitral annular diameter. Compared to the control group with severe ischaemic MR, the study group had shorter chordae, smaller leaflet tenting height, smaller tenting area, and smaller mitral annular diameter.

Conclusions: We report 10 subjects who underwent mitral valve surgery for severe MR attributable to pathologically short chordae. To the best of our knowledge, this is the first description of this mechanism of disease. Further work is needed to define the underlying factors that cause isolated mitral chordal disease.

Key words: mitral valve surgery, functional mitral regurgitation, chordal shortening.

\section{Introduction}

Mitral regurgitation (MR) that occurs in the absence of primary leaflet disease is known as function mitral regurgitation (FMR). FMR most commonly results from the left ventricular remodelling that occurs following

\author{
Corresponding author: \\ Wilbert S. Aronow MD, FACC, \\ FAHA \\ Department of Cardiology \\ Westchester Medical Centre \\ New York Medical College \\ Macy Pavilion \\ Room 141, Valhalla \\ NY 10595, USA \\ Phone: (914)-493-5311 \\ Fax: (914)-235-6274 \\ E-mail:wsaronow@aol.com
}


myocardial infarction or from primary cardiomyopathy [1-3]. Left ventricular (LV) enlargement often results in displacement of the papillary muscles, which can increase the distance from the papillary muscles to the mitral leaflets. When this distance is increased, chordal tethering of the mitral leaflets is frequently seen. Tethering limits leaflet closure and leaflet coaptation, causing regurgitation.

At our institution, we noted a cohort of subjects with severe MR, who had normal-appearing mitral valve leaflets and preserved LV size and function. On echocardiography these patients often appeared to have mitral leaflets with chordal tethering and restricted systolic closure despite having normal LV size. We hypothesised that primary shortening of the mitral chordae tendineae may be the mechanism of leaflet tethering and consequent regurgitation in this group. This case series describes subjects with FMR potentially attributable to chordal shortening, as supported by echocardiographic, intraoperative, and microscopic findings.

\section{Material and methods}

\section{Study group}

Our institution's cardiovascular database was queried for all subjects who underwent mitral valve surgery for regurgitation between 2006 and 2012 ( $n=658)$. For subjects in whom the aetiology of regurgitation was not well delineated in the echocardiography report, the images were reviewed by a senior echocardiographer (D.S.) to identify a potential mechanism. The study population consisted of subjects thought to have normal mitral leaflet appearance, normal left ventricular size and function, with a normal mitral annular dimension that subsequently had surgical repair or replacement. A comprehensive chart review was performed to record medical history, laboratory data, cardiac angiography, operative reports, and pathology reports. Subjects were excluded if operative valve inspection or gross pathological findings supported an obvious mechanism for valve regurgitation.

\section{Control groups}

Because echocardiographic measurements of mitral chordal length and leaflet tethering are not standardised, we selected two control groups to compare these measures with our study sample. The first control group consisted of normal echocardiograms. Ten subjects were selected from a sample of echocardiograms acquired in our laboratory over a 2-week period in December 2014. Studies were reviewed to for subjects with normal chambers sizes, normal ventricular function, and normal valve function. Subjects were includ- ed if they matched by age, sex, and body surface area to one of the study subjects. Ten additional control subjects with severe ischaemic MR were also selected. These controls were chosen from the same group of 658 subjects who underwent mitral surgery during the study period. Subjects were included in the ischaemic MR control group if the mitral leaflet appearance was normal, inferolateral LV infarction was present, and mitral leaflet tethering was reported. This control group was also matched by age, sex, and BSA to the study group.

\section{Echocardiography}

All study subjects had undergone transthoracic echocardiography (TTE) and transoesophageal echocardiography (TEE) within 60 days prior to surgery. Leaflets were assessed in both 2D and 3D planes for structural abnormalities. TTE was used to assess MR severity, LV size, LV wall motion, and LV ejection fraction. MR severity was classified by the American Society of Echocardiography (ASE) guidelines [2, 3]. Effective regurgitant orifice (ERO) and MR volume was assessed by PISA and VC methods using colour Doppler. Measurements of LV dimensions were performed at end diastole and systole in the parasternal long-axis view. LA volume and LV ejection fraction were obtained by biplane Simpson's method.

\section{Leaflet tethering parameters}

Mitral leaflet tethering parameters were measured by two different readers to allow assessment of inter-rater variability. Annular diameter, tenting height, and tenting area were obtained from TTE imaging in the PLAX view and end systole (Figure 1 A). Tenting height was measured as the distance between the coaptation point of mitral leaflets and the annular plane of the MV at mid-systole. Tenting area was measured as the area enclosed between the leaflets and the annular plane. Chordal length was assessed using TEE images and was measured from the papillary muscle tip to the leaflet margin on the P2 segment in both the transgastric long axis and mid-oesophageal four-chamber views. Posterior and apical papillary muscle displacement was measured using a method described by Dudzinski et al. [4-9].

\section{Statistical analysis}

Statistical analysis was done using Stata software, version 11.1 (College Station, TX). Normally distributed data were presented as the mean \pm standard deviation (SD). Non-normal data were presented as the median (interquartile range (IR)). Comparison of means was performed using the two-sample $t$-test. Comparison of categorical 

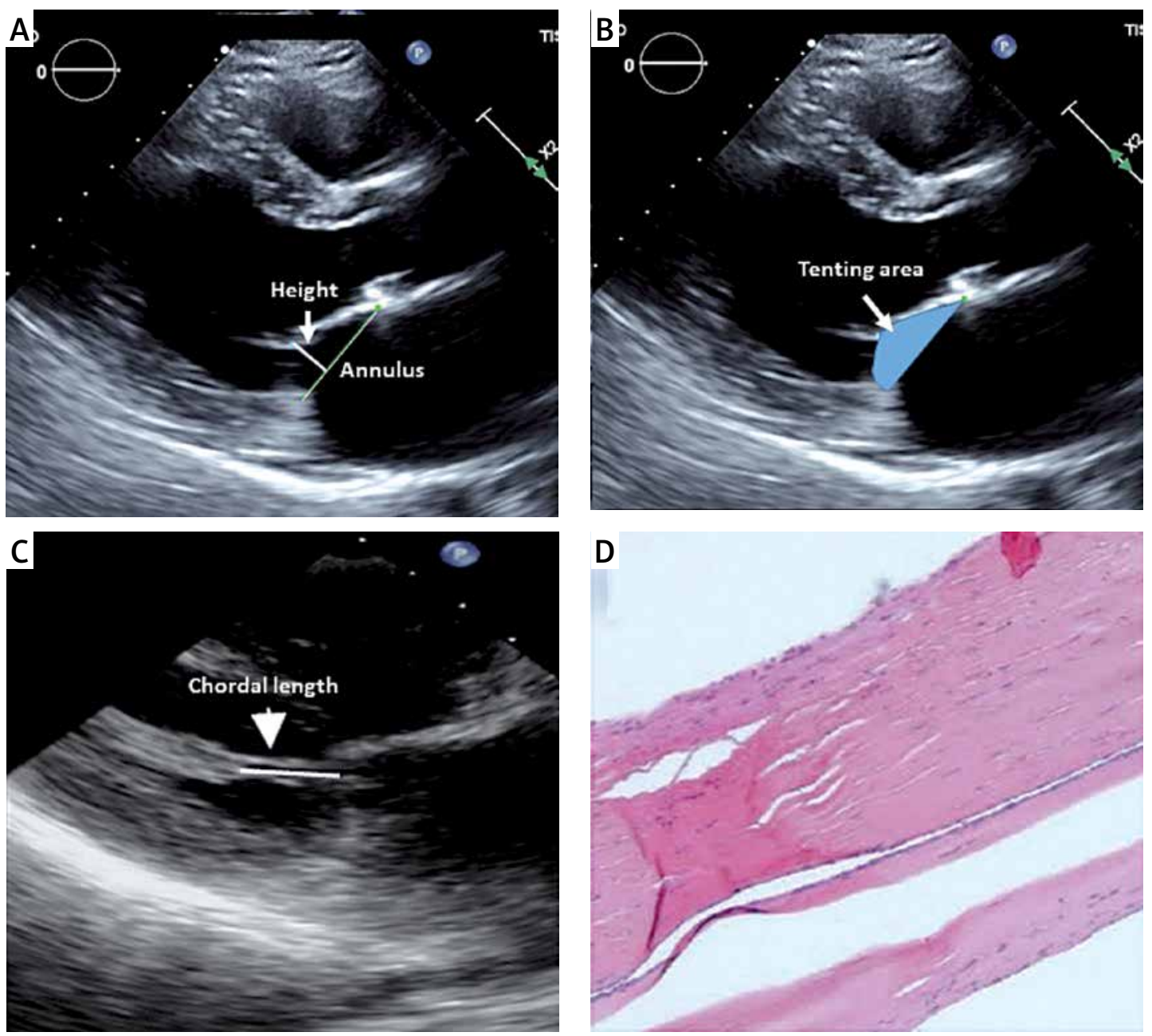

Figure 1. A, B - examples of mitral leaflet tethering parameters as measured on transthoracic echocardiography. C - an example of chordal measurement taken from transgastric view at $90^{\circ}$. D - haematoxylin and eosin staining of chordae tendineae removed at the time of mitral valve replacement. Pathological fibrosis is shown

data was performed using the chi-squared test. Comparison of medians was performed using the Mann-Whitney or Kruskal-Wallis tests as appropriate. $P$-values were considered significant if $<0.05$. Interobserver variability was compared using the mean and SD of the difference between the two readers' measurements. Paired $t$-test was used to compare measurements to check for measurement bias between readers.

\section{Results}

Patient demographics, echocardiographic, operative, and pathologic findings for the 10 included study subjects are presented in Tables I and II. Most of the cohort were female (9 of 10), with an average age of 63 years. None had history of rheumatic fever, endocarditis, malignancy, or other systemic inflammatory condition. Renal, hepatic, and thyroid function were normal in all study subjects. Electrolytes and troponin-T levels were also normal. Two subjects had single-vessel coronary disease, noted on perioperative angiography, and underwent bypass during the MV surgery. Six subjects underwent MV replacement, while the remainder had MV repair.

On surgical inspection chordal shortening or restriction was reported in six and chordal thickening or fibrosis was mentioned in two subjects. For those who underwent valve replacement (6 of $10)$, the excised portions of the leaflets demonstrated generally normal gross appearance. Four valves showed either focal fibrous or focal myxoid changes on histopathological inspection.

Chordal length and geometric indices are presented in Table II. Compared to normal controls, the study group had shorter chordae, increased mitral tenting height, and smaller mitral annular diameter. Compared to controls with severe ischaemic MR, the study group had shorter chordae, smaller leaflet tenting height, smaller leaflet tenting area, and smaller mitral annular diameter.

Using paired analysis, no difference was noted between readers for measurement of mitral tenting area $(p=0.9)$, tenting height $(p=0.8)$, and 


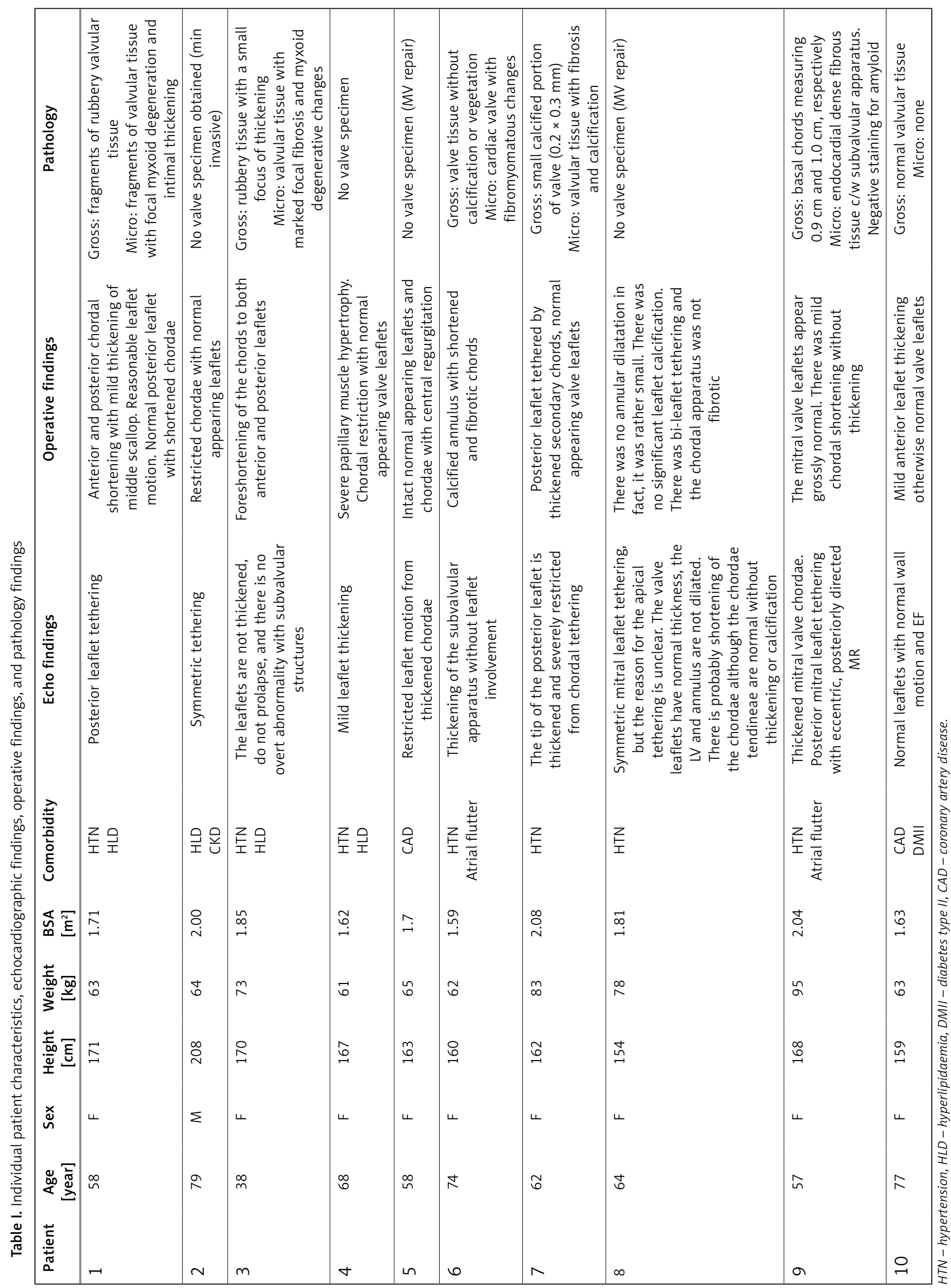


Table II. Individual patient echocardiographic chamber measurements, LV function, mitral regurgitation parameters, and mitral chordal length

\begin{tabular}{|lcccccccccc|}
\hline Patient & $\begin{array}{c}\text { LVEF } \\
(\%)\end{array}$ & $\begin{array}{c}\text { LAD } \\
{[\mathrm{cm}]}\end{array}$ & $\begin{array}{c}\text { LVESD } \\
{[\mathrm{cm}]}\end{array}$ & $\begin{array}{c}\text { LVEDD } \\
{[\mathrm{cm}]}\end{array}$ & $\begin{array}{c}\text { EDV } \\
{[\mathrm{ml}]}\end{array}$ & $\begin{array}{c}\text { ESV } \\
{[\mathrm{ml}]}\end{array}$ & $\begin{array}{c}\text { VC } \\
{[\mathrm{cm}]}\end{array}$ & $\begin{array}{c}\text { ERO } \\
{[\mathrm{cm}]}\end{array}$ & $\begin{array}{c}\text { Chordal } \\
\text { Length (AP4) } \\
{[\mathrm{cm}]}\end{array}$ & $\begin{array}{c}\text { Chordal } \\
\text { Length (SAX) } \\
{[\mathrm{cm}]}\end{array}$ \\
\hline 1 & 50 & 4.0 & 4.5 & 5.8 & 145 & 72 & 0.30 & 0.31 & 1.10 & 1.10 \\
\hline 2 & 60 & 5.3 & 3.3 & 4.6 & 88 & 35 & 0.80 & 0.38 & 1.20 & 1.30 \\
\hline 4 & 50 & 4.0 & 3.8 & 5.0 & 79 & 39 & 0.30 & 0.40 & 1.10 & 1.30 \\
\hline 5 & 60 & 5.1 & 3.6 & 5.5 & 95 & 38 & 0.60 & 0.58 & 1.10 & 1.00 \\
\hline 6 & 52 & 4.2 & 3.5 & 4.6 & 66 & 32 & 0.43 & 0.43 & 1.10 & 1.10 \\
\hline 7 & 52 & 5.0 & 2.6 & 4.2 & 56 & 27 & 0.44 & 0.44 & 1.30 & 1.40 \\
\hline 8 & 55 & 5.0 & 2.9 & 4.9 & 110 & 45 & 0.42 & 0.42 & 1.10 & 1.10 \\
\hline 9 & 55 & 5.3 & 3.1 & 3.4 & 106 & 48 & 0.30 & 0.30 & 1.20 & 1.30 \\
\hline 10 & 60 & 5.1 & 2.3 & 3.4 & 66 & 26 & 0.20 & 0.20 & 1.20 & 0.88 \\
\hline
\end{tabular}

LVEF - left ventricular ejection fraction, LAD - left atrial diameter, LVESD - left ventricular end systolic dimension, LVEDD - left ventricular end diastolic dimension, EDV - end diastolic volume, ESV - end systolic volume, VC - vena contracta, ERO - effective regurgitant orifice.

chordal length $(p=0.9)$. Measurement of mitral annular diameter was different between readers (3.8 \pm 0.3 vs. $3.4 \pm 0.3 \mathrm{~cm}, p=0.04)$.

\section{Discussion}

This report describes a series of subjects who underwent surgery for mitral regurgitation despite having normal leaflets, normal annular dimension, and normal LV size. We propose that the mechanism of regurgitation in this group is primarily related to shortened mitral chordae tendineae with consequent mitral leaflet tethering. To our knowledge this represents the first description of chordal shortening as an isolated mechanism for mitral regurgitation. Our results showed that chordal length was significantly shorter in the study population compared to healthy controls and also compared to those with ischaemic FMR.

The control subjects with ischaemic functional MR in our study had similar chordal length to those reported in prior works. In contrast, the study subjects in our report had shorter chordal length compared to historical controls (Figure 2) [10, 11].

Comparison of mitral geometry between the study group and controls revealed several important findings. Whereas increased leaflet tethering (tenting height toward the apex) was notable between the study subjects and the normal controls, tenting in the study group was not as pronounced as was observed in the controls with ischaemic FMR. In addition, we found that the study group had smaller mitral annuli compared to both the normal controls and the controls with ischaemic FMR. Although the measured tenting area was not demonstrated to be increased in the study group compared to normal controls, we suspect that this was the result of measurement error, because apical tenting was evident with subjective visual inspection in all of the study subjects (Figure 1).

Histological evaluation of our study population was utilised to identify a potential common mechanism of the shortened chordae. In subjects for whom tissue analysis was available, we found mild abnormalities in the subvalvular apparatus; however, no common pathognomonic appearance could be identified. The most common abnormality noted was myxomatous change, but this was seen with approximately the same prevalence as in the general population [12].

Our study has several important limitations. One limitation is the lack of in vivo intraoperative measurement of the chordae. Because the study subjects were enrolled retrospectively, this measurement could not be obtained. However, use of TEE to measure the chordal length in the beating heart had good interobserver agreement in our

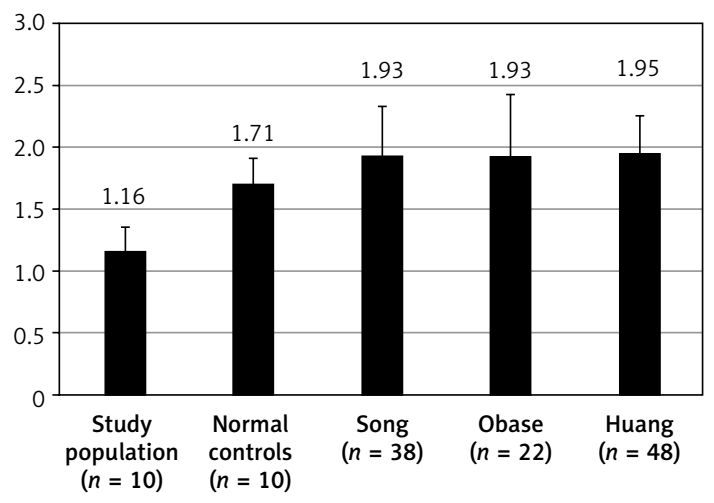

Figure 2. Chordal lengths measured in our study population, in our normal controls, and in several other published series 
study and showed good agreement with historical controls. The histological evaluation was limited by the retrospective analysis because we were not able to stain the fixed slides post hoc. Use of 3D TEE would also have been helpful to measure mitral leaflet area. Since mitral leaflet remodelling is a common feature in FMR, examination for this phenomenon in the study group would help give more insight into the mechanism of regurgitation [13-15]. Future studies are needed with a larger dataset and prospective planning of tissue analysis to better characterise our findings.

\section{Conflict of interest}

The authors declare no conflict of interest.

\section{References}

1. Otto CM. Textbook of Clinical Echocardiography. Saunders/Elsevier, Philadelphia 2009.

2. Zoghbi W, Enriquez-Sarano M, Foster E, et al. Recommendations for evaluation of the severity of native valvular regurgitation with two-dimensional and Doppler echocardiography. J Am Soc Echocardiogr 2003; 16: 777-802.

3. Lang RM, Bierig M, Devereux RB, et al. Recommendations for chamber quantification: a report from the American Society of Echocardiography's Guidelines and Standards Committee and the Chamber Quantification Writing Group, developed in conjunction with the European Association of Echocardiography, a branch of the European Society of Cardiology. I Am Soc Echocardiogr 2005; 18: 1440-63.

4. Dudzinski DM, Hung J. Echocardiographic assessment of ischemic mitral regurgitation. Cardiovasc Ultrasound 2014; 12: 46.

5. Kim K, Kaji S, An Y, et al. Mechanism of asymmetric leaflet tethering in ischemic mitral regurgitation: 3D Analysis with multislice CT. JACC Cardiovasc Imaging 2013; 5: $230-2$.

6. Obase K, Weinert L, Hollatz A, et al. Leaflet-Chordal relations in patients with primary and secondary mitral regurgitation. J Am Soc Echocardiogr 2015; 28: 1302-8.

7. Song JM, Fukuda S, Kihara T, et al. Value of mitral valve tenting volume determined by real-time three-dimensional echocardiography in patients with functional mitral regurgitation. Am J Cardiol 2006; 98: 1088-93.

8. Sadeghpour A, Abtani F, Kiavar M, et al. Echocardiographic evaluation of mitral geometry in functional mitral regurgitation. J Cardiothorac Surg 2008; 3: 54.

9. Obase K, Jeevanandam V, Saito K, et al. Visualization and measurement of mitral valve chordae tendineae using three-dimensional transesophageal echocardiography from the transgastric approach. J Am Soc Echocardiogr 2015; 28: 449-54.

10. Huang $\mathrm{H}$, Xie X, Fei $\mathrm{H}$, et al. Real-time three-dimensional transesophageal echocardiography to predict artificial chordae length for mitral valve repair. J Cardiothorac Surg 2013; 8: 137 .

11. Song JM, Kim JJ, Ha TY, et al. Basal chordae sites on the mitral valve determine the severity of secondary mitral regurgitation. Heart 2015; 101: 1024-31.

12. Turri M, Thiene G, Bortolotti U, Mazzucco A, Gallucci V. Surgical pathology of disease of the mitral valve, with special reference to lesions promoting valvar incompetence. Int J Cardiol 1989; 22: 213-9.

13. Saito $\mathrm{K}$, Okura $\mathrm{H}$, Watanabe $\mathrm{N}$, et al. Influence of chronic tethering of the mitral valve on mitral leaflet size and coaptation in functional mitral regurgitation. JACC Cardiovasc Imaging 2012; 5: 337-45.

14. Debonnaire P, Al Amri IA, Leong DP, et al. Leaflet remod eling in functional mitral valve regurgitation: characteristics, determinants and relation to regurgitation severity. Eur Heart I Cardiovasc Imaging 2015; 16: 290-9.

15. Beaudoin J, Handschumacher MD, Zeng X, et al. Mitral valve enlargement in chronic aortic regurgitation as a compensatory mechanism to prevent functional mitral regurgitation in the dilated left ventricle. J Am Coll Cardiol 2013; 61: 1809-16. 